Conclusions Significant cost savings can be made, and endoscopy capacity generated, by discontinuing colonoscopy surveillance for LRAs. In the increasingly financially constrained NHS environment this approach should be explored, particularly in patients who are eligible for participation in the BCSP.

\section{PTU-105 AUTOMATED, ALGORITHM BASED EXTRACTION OF BARRETT'S SURVEILLANCE METRICS FROM NATURAL LANGUAGE TEXT IS RELIABLE}

\begin{abstract}
${ }^{1}$ Sebastian Zeki*, ${ }^{2}$ Richard Hackett, ${ }^{1}$ Jason Dunn, ${ }^{1}$ Aaron Bancil, ${ }^{3}$ Sean Preston, ${ }^{3} J o a n n e$ Chin-Aleong, ${ }^{4}$ Jonathan Brown, ${ }^{2}$ Stuart McDonald. 'Guy's and St Thomas' NHH Trust, London, UK; ${ }^{2}$ Tumour Biology, Bart's Cancer Institute, London EC1M, UK; ${ }^{3}$ Barts and the London School of Medicine and Dentistry, Queen Mary University of London, London, UK; ${ }^{4}$ Gloucestershire Royal Hospital, Gloucester, UK
\end{abstract}

\subsection{6/gutjnl-2019-BSGAbstracts.464}

Introduction Patients with Barrett's oesophagus (BE) undergo regular endoscopic surveillance with a view to earlier oesophageal adenocarcinoma detection. Quality monitoring of this programme relies on manual extraction of elements from pathology and endoscopic semi-structured free text reports. Manual extraction is laborious and a significant hindrance to robust, large scale and reproducible quality monitoring.

EndoMineR, a package written in R, (a free, open source computational language) has been developed specifically to automate the extraction of data from endoscopic and associated pathology reports ${ }^{1}$. It contains functions to clean, format and extract elements from free text and perform quality metrics for a range of conditions including in BE.

Aim We assessed the accuracy of the BE extraction algorithms for both endoscopic and pathological elements for $\mathrm{BE}$ on pathology data only as it is the 'worst case scenario' input data, using the EndoMineR package. The functions being assessed were: 1. The extraction of a Prague score, 2. The extraction of the worst pathology grade, 3. The site of biopsied tissue, 4. The site and type of any therapy in the upper GI tract.

Methods Ethics was approved (IRAS number). 60 patient episodes between 14 January 2016 and 30 March 2016 with full text pathology data only were acquired from 8 departments in central London as a training set. Validation was performed on a further 100 pathology reports. The therapy algorithm was performed on a further 100 reports.

Abstract PTU-105 Table 1 Sensitivity, specificity, positive and negative predictive values of each of the functions being assessed

\begin{tabular}{lllll} 
Abstract PTU-105 & Table 1 & & & \\
\hline & Sensitivities & Specificities & PPV & NPV \\
\hline MStage & 97.67 & 78.57 & 0.97 & 0.85 \\
CStage & 94.23 & 97.92 & 0.98 & 0.94 \\
Worst & 97.83 & 62.5 & 0.97 & 0.71 \\
Pathology & & & & \\
PathologySite & 88 & 100 & 1 & 0.89 \\
TherapySite & 95.92 & 82.35 & 0.84 & 0.95 \\
\hline
\end{tabular}

Results Reports were written by 11 different pathologists. The readability index of all the text, using the Fleisch-Kincaid readability index was 11.7 (sd:1.22) indicating an average grammatical complexity. Sensitivity was excellent for all algorithms especially given the difficult input text (Table 1). A reduction in specificity in the detection of worst pathology occurred because of dual reporting of colonoscopy and gastroscopy tissue which also affected the sensitivity of the Pathology Site detection. A variability in how intestinalisation was reported also affected the specificity.

Conclusion

- Reproducible extraction can be done from semi-structured text.

- Further improvements using parts of speech tagging and term mapping will improve the results.

- Such data extraction will allow for upstream automation of quality monitoring, governance and novel metrics.

\section{REFERENCES}

1. Zeki S of Open Source, (2018). EndoMineR for the extraction of endoscopic and associated pathology data from medical reports. Journal Software;3(24):701.

\section{PTU-106 BARRETT'S OESOPHAGUS - ARE WE FOLLOWING THE GUIDELINES?}

${ }^{1}$ Mina Soliman, ${ }^{2}$ Elmuhtady Said, ${ }^{2}$ Kapil Kapur. 'ST George University of London, London, UK; ${ }^{2}$ Barnsley Foundation Trust Hospital, Barnsley, UK

\subsection{6/gutjnl-2019-BSGAbstracts.465}

Introduction Barrett's oesophagus (BO) affects $1-5 \%$ of reflux patients and has a premalignant potential. Several guidelines $1,2,3$ to optimise surveillance are tailored towards discovering dysplasia and hence allow treatment and prevention of cancer. Aim and Methods We aim to evaluate compliance of BO surveillance with the current published guidelines in our centre between June 1st 2017 to May 31st 2018.

Retrospective database search was done using the built-in audit tool of Infoflex v.5 reporting system. Surveillance details were added to an excel spreadsheet against criteria extracted from both the British Society of Gastroenterology and the European Society guidelines. Histology and advice on proposed future surveillance were also reviewed.

Results 164 BO reports were found during the study period. $78 / 164$ (48\%) patients were undergoing surveillance and 86/ 164 (52\%) were newly diagnosed.

In the surveillance group: 54/78 (69\%) were males, mean age 67(4-0) and 24/78 (31\%) females, mean age 60 (range 56 ). Prague classification (PC) was correctly used in $70 / 78$ (89\%). Seattle protocol for biopsy was followed in 55/78 (70\%). Chromoendoscopy was used in 26/78 (33\%). Inspection time was recorded only in $2 / 78$ (2.5\%). In two cases(2/ 78 ), visible lesions were found and described according to the clock face and its distance from incisors. Intestinal metaplasia was confirmed on histology in 72/78 (92\%). Recommended surveillance frequency was consistent with the guidelines in $64 / 72(88 \%)$ of BO without dysplasia but in all 3 cases with dysplasia. In the group presenting to symptomatic service where a new diagnosis of BO was found: 59/86 (68\%) had PC correctly listed. Of the remaining 27/86, all but one were on a surgical list. Additionally, only 41/86 (47\%) of the suspected new BO were biopsied. Reasons for not taking biopsies were listed in 10 cases only.

Conclusions There is significant variability in the execution and surveillance of BO. The correct use of Seattle protocol, chromoendoscopy and recording the time spent inspecting the $\mathrm{BO}$ are all important service improvements that could be 
achieved through dedicated lists, and regular audits. Including summary of the guidelines in endoscopists'induction might also improve new $\mathrm{BO}$ diagnosis.

\section{REFERENCES}

1. Fitzgerald, et al. BSG guidelines on the diagnosis and management of Barretts oesophagus. Gut. 2013;63(1):7-42.

2. Beg, et al. Quality standards in upper gastrointestinal endoscopy: a position statement of the BSG and Association of Upper Gastrointestinal Surgeons. Gut. 2017; 66(11): 1886-99.

3. Bisschops, et al. Performance measures for upper gastrointestinal endoscopy: a European Society of Gastrointestinal Endoscopy (ESGE) Quality Improvement Initiative. Endoscopy. 2016; 48(09): 843-64.

\section{PTU-107 VIDEO OUTPATIENT SERVICES: CAN PATIENT CHOICE IN HOW TO ATTEND BE A SUCCESS?}

${ }^{2}$ Philip Cannon, ${ }^{2}$ Craig Fraser, 'Wilson Siu, 1,2 John Thomson*. 'NHS Grampian, Aberdeen, UK; ${ }^{2}$ University of Aberdeen, Aberdeen, UK

10.1136/gutjnl-2019-BSGAbstracts.466

Introduction Recent technological advancements have created the opportunity to radically transform how outpatient services are delivered. Citizens with chronic relapsing/remitting disease such as Inflammatory Bowel Disease (IBD) are often required to attend regular follow up clinic appointments with the associated travel and time off work creating considerable effects on carbon emissions and the wider economy.

Methods As part of a wider program of work to establish a patient focussed outpatient service a pilot study was instigated where all patients were offered the option of attending their IBD appointment using a video clinic platform (Attend Anywhere $\left.{ }^{\circledR}\right)$. This abstract describes the evaluation phase of patient initiated video appointments from patient and clinician viewpoints. The uptake of video appointments was recorded from the Patient Administration System along with demographics of the tele-medicine (video) and anchi-medicine (traditional clinic) groups. Patient perspectives were captured by an online questionnaire presented to them on concluding the video appointment and clinician perspective was captured with the clinic documentation.

Results During the 7 month study period 194 (12\%) opted for a video appointment of which 85 (41\%) completed the patient questionnaire. There were no significant differences in demographics or proportion of did not attend (DNA) appointments between the groups. However, $77 \%$ of the video group were noted to have minimal symptoms. In the patient questionnaire, 85 (100\%) indicated they would use video again, 80 (94\%) found it more convenient than attending the hospital and $54(64 \%)$ indicated they had not had to take time off work. The average travel saved was 117 miles per appointment. However, 33\% experienced technical issues during the consultation.

The clinician data indicated that $80 \%$ of the video consultations had been entirely successful with the majority of unsuccessful video consultations being completed by telephone. Two patients required a further anchi-medicine appointment for examination and three required to attend for blood tests only available at the clinic.

Conclusions In line with the increasing use of technology in our society, this study has indicated that the patient can successfully make the choice of how to attend the clinic with little, if any, impact on the wider clinic service. However, such changes must be planned and prepared for so that other clinic processes such as booking and blood investigations procedures can be accommodated in the new clinic model. As with all technology adoption there is a curve of uptake, as the technology underpinning video clinics becomes mainstream it is anticipated that video will become an increasingly popular method of outpatient review technology with patients and clinicians.

\section{PTU-108 PROSPECTIVE COHORT TO IDENTIFY FACTORS ASSOCIATED WITH DIAGNOSTIC DELAY IN PATIENTS WITH INFLAMMATORY BOWEL DISEASE}

\footnotetext{
1,2 Gareth Walker, ${ }^{1,2}$ Simeng Lin, ${ }^{1,2}$ Amanda Thomas, ${ }^{1,2}$ Neil Chanchlani, ${ }^{2}$ Lucy Moore, ${ }^{1,2}$ Peter Hendy, ${ }^{1,2}$ Neel Heerasing, ${ }^{3}$ Harry Green, ${ }^{1,2}$ Sean Mole, ${ }^{2}$ Claire Bewshea, 1,2 James Goodhand, 1,2 Nick Kennedy, 1,2Tariq Ahmad. 'Royal Devon and Exeter Hospital, Exeter, UK; ${ }^{2}$ University of Exeter, IBD Pharmacogenetics, Exeter, UK; ${ }^{3}$ University of Exeter Medical School, Genetics of Complex Traits, Exeter, UK
}

\subsection{6/gutjnl-2019-BSGAbstracts.467}

Background International cohort studies have previously identified Crohn's disease (CD), ileal disease, smoking, and age $(<40$ years old) as factors associated with a delay in diagnosis of patients with inflammatory bowel disease (IBD). Currently, there is a paucity of data looking at the factors influencing diagnostic delay specific to a UK population, where healthcare system is free at point-of-access. Hence, we conducted a prospective observational cohort study of patients referred to secondary care between January 2014 to December 2017.

Methods In total, 163 patients between the age of 18 and 46 years who first presented to their general practitioner (GP) with gastrointestinal symptoms from January 2014 were included in this study. Patients above the age of 46 were excluded due to the increased risk of colorectal cancer with increasing age. This was also the upper age limit recommended for faecal calprotectin use in the investigation of suspected IBD. In addition to baseline demographic data, our main outcome measure was time to overall diagnosis including time from onset of symptoms to GP presentation (patient delay), time of GP presentation to referral (primary care delay), and time of referral to diagnosis (secondary care delay).

Results The median time to diagnosis was 6.7 months [IQR 3.3-14.1], with no significant difference in time to diagnosis for IBD sub-types [CD, 9.8 months [IQR 5.5-18.5]; IBDUnclassified, 7.0 months [IQR 4.5-8.5] and ulcerative colitis (UC), 5.2 months [IQR $2.9-12.3$ ] $(\mathrm{p}=0.56$ )]. The median time it took patients to present to their GP was 3.0 months [IQR 1.4-6.0]; median time for GP to refer to a gastroenterologist was 0.6 months [IQR 0.2-1.7]; and the median time from GP referral to diagnosis was 1.5 months [IQR 0.8-2.5]. On multivariable analysis, rectal bleeding (OR 0.33, 95\% CI $0.15-0.71, \mathrm{p}=0.005$ ) and abdominal pain (OR 2.49; 95\% CI $1.13-5.89, \mathrm{p}=0.029)$ was negatively and positively associated with being in the upper quartile of patient delay. Urgent GP referrals (OR 0.14; 95\% CI 0.05-0.36, p < 0.001 ) and triage by surgeons (OR 5.61; 95\% CI 2.29-14.38, $\mathrm{p}<0.001)$ had a negative and positive association with being in the upper quartile of secondary care delay, respectively. The use of faecal calprotectin or being triaged straight-to-test did not reach statistical significance.

Conclusion Referrals triaged urgently and by a gastroenterologist were associated with a reduction in secondary care diagnostic delay. Adopting a combination of primary care faecal 